\title{
Substituted septithiophenes with end groups of different size: Packing and frustration in bulk and thin films
}

Wim H. de Jeu, Khosrow Rahimi, Ulrich Ziener, Roman Vill, Eva M. Herzig, Peter MüllerBuschbaum, Martin Möller and Ahmed Mourran*

[*] Dr. A. Mourran, Prof. W. H. de Jeu, Dr. K Rahimi, Prof. M. Möller, DWI-Leibniz Institute for Interactive Materials,

Forckenbeckstrasse 50, D-52056 Aachen, Germany,

E-mail: mourran@dwi.rwth-aachen.de

Prof. U. Ziener, Roman Vill,

University of Ulm, Institute of Organic Chemistry III,

Albert-Einstein-Allee 11, D-89081 Ulm, Germany,

Dr. E. M. Herzig, Prof. P. Müller-Buschbaum,

Technische Universität München, Lehrstuhl für Funktionelle Materialien,

James-Franck-Strasse 1, Garching, 85478, (Germany)

Keywords: Oligothiophenes, Liquid crystal phases, Bulk structures, Monolayers, AFM, GIWAXS 


\begin{abstract}
We report on three different liquid crystalline compounds with a central septithiophene core and alkylated end groups of strongly increasing bulkiness. In principle, the thiophene cores prefer to pack parallel to optimize their $\pi-\pi$ interactions, which becomes sterically impossible for the bulkier end groups. Using x-ray diffraction, we find that the way out of this packing dilemma is toward liquid-crystal phases of higher dimensionality in the order smectic $\rightarrow$ columnar $\leftrightarrow$ bicontinuous cubic. For the smectic phase, packing in a monolayer is no problem, for the other ones packing considerations become more stringent in films due to the boundaries. Surface $\mathrm{x}$-ray techniques and atomic force microscopy indicate an appreciable difference between monolayer and three-layer films, in which the monolayers appear to escape from packing frustration by generating superstructures. We propose a basic structure of columns parallel to the substrate that provides a compromise between preserving some $\pi-\pi$ interactions and packing the bulky alkyl groups.
\end{abstract}




\section{Introduction}

Conventional liquid crystals involve essentially a nematic phase (elongated molecules that combine orientational order with a liquid structure) and various types of smectic phase with an additional one-dimensional density modulation. The molecular design principles of these systems are well-known: the basic concept is a molecule with a rigid rod-like anisometric core and flexible end chains. In this way a typical combination of order and mobility can be obtained, which is a prerequisite for applications like liquid crystal displays. Chirality induces additional symmetry elements. Rotational order of the anisometric segments can lead to polar order and/or achiral symmetry breaking into bent-core systems. As a result a myriad of liquidcrystal (LC) phases has been generated. ${ }^{1-3}$ Supramolecular chemistry has modified and complicated this picture considerably by interconnecting different mesogens (dimesogens, oligomesogens, dendrimers, polymers). This broad context sets the framework for problems of packing and frustration in anisotropic conjugated systems in dependence of their structure. In the present work we consider some oligomers of thiophenes with rather differently shaped substituents, both in bulk and in thin films.

A defining structural property of oligothiophenes ${ }^{4}$ is the transverse $\pi-\pi$ coupling between neighbouring conjugated cores. ${ }^{5}$ The coupling can be influenced by the end substituents. A substituent will add or withdraw density from the $\pi$ system of the conjugated core, thus changing the electrostatic interaction. This effect is nonlocal and indirect. Furthermore, there might be a direct interaction due to an altered polarity of the linking $\sigma$ bond aromaticsubstituent. ${ }^{5}$ In addition to the basic forces (Vander Waals attraction and steric repulsions) these effects are expected to influence the structure and packing and thus any LC behaviour. Combining elongated molecular units in a parallel way requires a certain matching of their transverse dimensions to reduce packing frustration. This can be reached, for example, by either tilting one unit with respect to the other or by (partial) overlapping (interdigitation). ${ }^{6,7}$ In such a situation a fundamental difference arises between bulk and thin-film structures: Neighbouring overlapping structures in the bulk have no equivalent in a monolayer. In addition specific surface interactions will come into play. ${ }^{8}$ Any alternation of the neighbourneighbour packing due to the surface is expected also to influence the $\pi-\pi$ interactions between the cores.

LC order in oligothiophenes provides a means to control the growth of uniform structures as required for applications of their intrinsic hole mobility. ${ }^{6,9-12}$ Various substituents have been introduced symmetrically at the $\alpha, \omega$ positions of the thiophene core in order to reduce the melting point and promote LC phases. ${ }^{13}$ The phase behaviour - including the transition 
temperatures determining the LC window - originates from a delicate balance between length and structure of both core and substituents. ${ }^{11,14-19}$ Previously, we have shown that LC order is favoured by a branched alkyl substituent, 2-octyldodecyl, at the $\alpha, \omega$ positions of septithiophene (7T, see Fig. 1) ${ }^{6}$, which shows a smectic LC phase between 166 and $171^{\circ} \mathrm{C}$. In a three-dimensional (3-D) crystal the packing frustration due to the branched aliphatic chains is alleviated by interdigitation of the alkyl chains and tilt of the thiophene cores. As a next step asymmetry has been introduced by attaching different kinds of substituents. This leads to additional frustration in the packing of the molecules, promoting LC phases. Recently, this work has been extended from asymmetrically substituted quaterthiophene ${ }^{20-22}$ to sexithiophene. $^{7}$

$7 \mathrm{~T}$

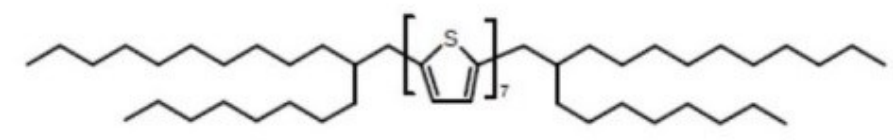

7TH

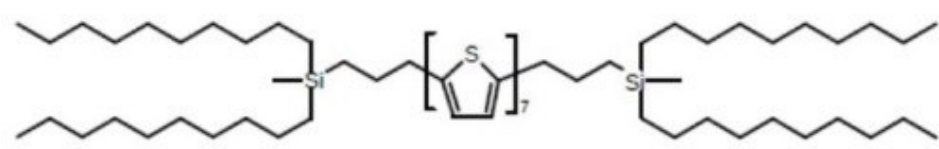

$7 \mathrm{TJ}$

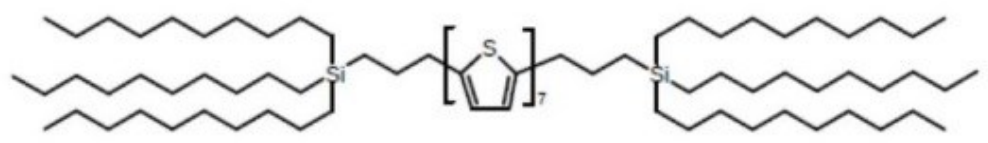

Figure 1. Molecular structure of the compounds investigated.

Considerable structure variation of symmetrically substituted oligothiophenes has been realized, ${ }^{16,17,23,24}$ for which the melting point, the self association in solution, and the hole mobility in monolayers could be rationalized in terms of a simple bulkiness parameter. ${ }^{24}$ In the present work we investigate in depth some of these materials. In particular we compare 7T with two other substituted septithiophenes indicated as $7 \mathrm{TH}$ and $7 \mathrm{TJ}$, respectively (see Figure 1) that have increasingly bulky end substituents. For the smectic phase of 7T the difference in average cross section between the thiophene core and the terminal alkyl chains leads to a tilt angle of $58^{\circ}$ between the core and the chains. ${ }^{6}$ For the other compounds such a simple solution to the packing problem is not possible anymore. Instead we find highly ordered liquid-crystalline phases (columnar and gyroid cubic).

Uniformity of the alignment of the molecules in monolayers is of great practical importance. Optimizing the uniformity of a crystalline thin film involves the delicate point of decoupling of lateral (two-dimensional: 2-D) growth from that perpendicular to the substrate 
leading to multilayers. ${ }^{25}$ It has been assumed that molecules interacting weakly on dielectric substrates like $\mathrm{SiO}_{2}$ and the presence of a mesomorphic intermediate state facilitates and extends the range of alignment. ${ }^{26-28}$ Limitations in the use of polycrystalline thin films arise from structural defects as well as from grain boundaries. Unsubstituted oligothiophenes are usually crystalline and the $\pi-\pi$ coupling between the edge-to-face alignment of the thiophene rings yields 2-D electronic interactions. The combinations of surface interactions with LC order of oligothiophenes can potentially provide self-healing of structural defects, leading to the formation of large ordered domains. ${ }^{12}$ Smectic LC order is most appealing because of its compatibility with a 2-D monolayer structure ${ }^{6,7}$ and has been investigated already for our reference compound $7 \mathrm{~T}$. $^{5}$ From the bulk LC structures of the compounds $7 \mathrm{TH}$ and $7 \mathrm{TJ}$, it is not obvious how the monolayers will organize. In the present work the bulk structures will be compared with those in thin films, in particular also monolayers, using both surface x-ray techniques and atomic force microscopy. The results indicate an appreciable difference between monolayer and three-layer films, the structure of the latter tending already to the bulk one.

Table 1. Phase transition temperatures $\left({ }^{\circ} \mathrm{C}\right)$ from DSC and from optical microscopy. $\mathrm{Cr}$, LC and I denote a crystalline, liquid-crystalline and isotropic phase, respectively. The transition enthalpy in $\mathrm{Jg}^{-1}$ is given in brackets.

\begin{tabular}{|c|c|c|}
\hline compound & DSC (heating) & microscopy (cooling) \\
\hline \multirow[t]{2}{*}{$7 \mathrm{~T}$} & $\mathrm{Cr} \rightarrow 63 \rightarrow \mathrm{Cr} \rightarrow 166 \rightarrow \mathrm{LC} \rightarrow 171 \rightarrow \mathrm{I}$ & $\mathrm{I} \rightarrow 171 \rightarrow \mathrm{LC} \rightarrow 166 \rightarrow \mathrm{Cr} \rightarrow<63 \rightarrow \mathrm{Cr}$ \\
\hline & $(9.4)$ & \\
\hline \multirow[t]{2}{*}{$7 \mathrm{TH}$} & $\mathrm{Cr} \rightarrow 89 \rightarrow \mathrm{LC} 2 \rightarrow 127 \rightarrow \mathrm{LC} 1 \rightarrow 177 \rightarrow \mathrm{I}$ & $\mathrm{I} \rightarrow 172 \rightarrow \mathrm{LC} 1 \rightarrow 130 \rightarrow \mathrm{LC} 2 \rightarrow<90 \rightarrow \mathrm{Cr}$ \\
\hline & $(2.27)$ & \\
\hline \multirow[t]{2}{*}{ 7TJ } & $\mathrm{Cr} \rightarrow 38 \rightarrow \mathrm{LC} \rightarrow 127 \rightarrow \mathrm{I}$ & $\mathrm{I} \rightarrow 121 \rightarrow \mathrm{LC} \rightarrow<40 \rightarrow \mathrm{Cr}$ \\
\hline & (13.6) & \\
\hline
\end{tabular}

\section{Bulk results}

\subsection{Materials and phase transitions}

The phase transition temperatures of the various compounds are given in Table 1. The original results from differential scanning calorimetry can be found in the Supplementary Information as Figs. S1 and S2. The LC phase of 7T is known to be smectic with a large transition heat at the melting point of $166^{\circ} \mathrm{C} .{ }^{6}$ From their heats of transition, the LC phases of 
the other two compounds, 7TH and 7TJ, seem to be higher ordered like columnar or bicontinuous cubic.

\subsection{X-ray characterization of the LC phases}

In principle $\mathrm{x}$-ray scattering should give conclusive information about the phase behaviour. First we return to the earlier results for $7 \mathrm{~T}^{6}{ }^{6}$ Below the transition to the isotropic phase we note at relatively small angles a smectic LC phase with a period of $3.4 \mathrm{~nm}$ (see Fig 2 at $168.5^{\circ} \mathrm{C}$ ). A diffuse outer ring around $0.34 \mathrm{~nm}$ (not shown) indicates 'liquid' layers. A closer inspection around the onset of this phase indicates a rather curious behaviour. Upon approaching the oriented smectic phase from below, we note that at $163-163.5^{\circ} \mathrm{C}$ the smectic ring (at $3.4 \mathrm{~nm}$ ) comes up next to the existing signal of a low-temperature lamellar structure (outer ring corresponding to $3.0 \mathrm{~nm}$ ). At higher temperatures the latter ring splits into a series of spots $\left(164^{\circ} \mathrm{C}\right.$ in Fig. 2$)$ before disappearing, and leaving only the high-temperature smectic phase. We assume that in fact six spots are present, two of which are hidden below the main smectic peak, indicating hexagonal symmetry. We note a strong parallelism with x-ray pictures of perforated lamellae in block copolymers. ${ }^{29}$ Nevertheless the final high-temperature phase at $168.5^{\circ} \mathrm{C}$ seems definitely smectic with a single period. Hence we suppose that a transition state between crystal and smectic is involved that deserves further investigation.
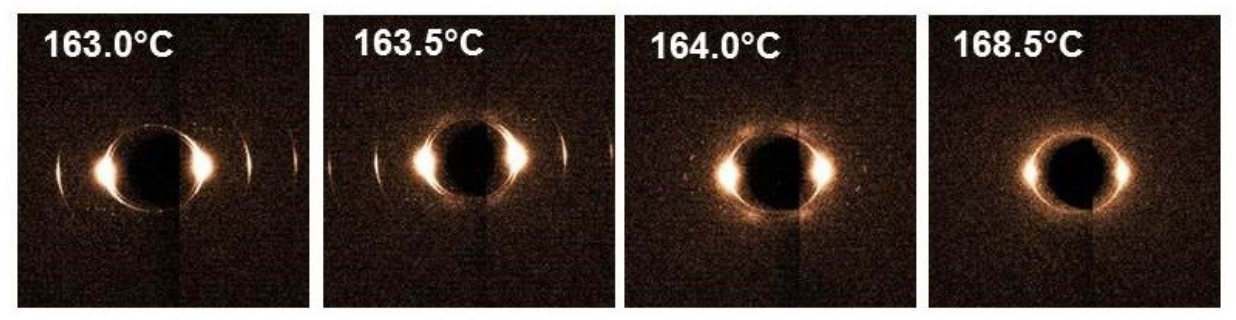

Figure 2. Onset of the smectic phase of $7 \mathrm{~T}$ around $166^{\circ} \mathrm{C}$. The smectic period is $3.4 \mathrm{~nm}$ while a diffuse outer ring (not shown) around $0.34 \mathrm{~nm}$ indicates disordered layers.

Figure 3 gives diffractograms of the two LC phases of 7TH with the peak positions in Table 2. These data are azimuthal averages of unoriented (powder) pictures. In addition a diffuse outer ring is observed (not shown), still at a spacing around $0.34 \mathrm{~nm}$. Clearly these compounds show no simple smectic phases anymore. Literature results ${ }^{30-32}$ indicate that with increasing volume fraction of lateral alkyl chains, the interface curvature between the nanosegregated regions of the thiophene cores and the flexible chains should increase. Such a structural variation can give rise to a transition from smectic through columnar to cubic phases, similar to the mesomorphism of amphiphiles. Guided by this principle, we note that 
the LC2 phase has reflections close to those of the bicontinuous gyroid phase (Ia3d). Then the peak positions are ordered according to ratios $\sqrt{ } 3: 2: \sqrt{ } 7: 2 \sqrt{ } 2: 4$, leading to the numbers in the fourth column of Table 2 under LC2. These values are close to the observed ratios in the third column with the exception of peak no.3. From Fig. 3 we note that this peak is at least a doublet, creating uncertainties about its origin. Hence we tentatively still conclude to a gyroid phase with a lattice parameter $a=3.4 \mathrm{~nm}$.
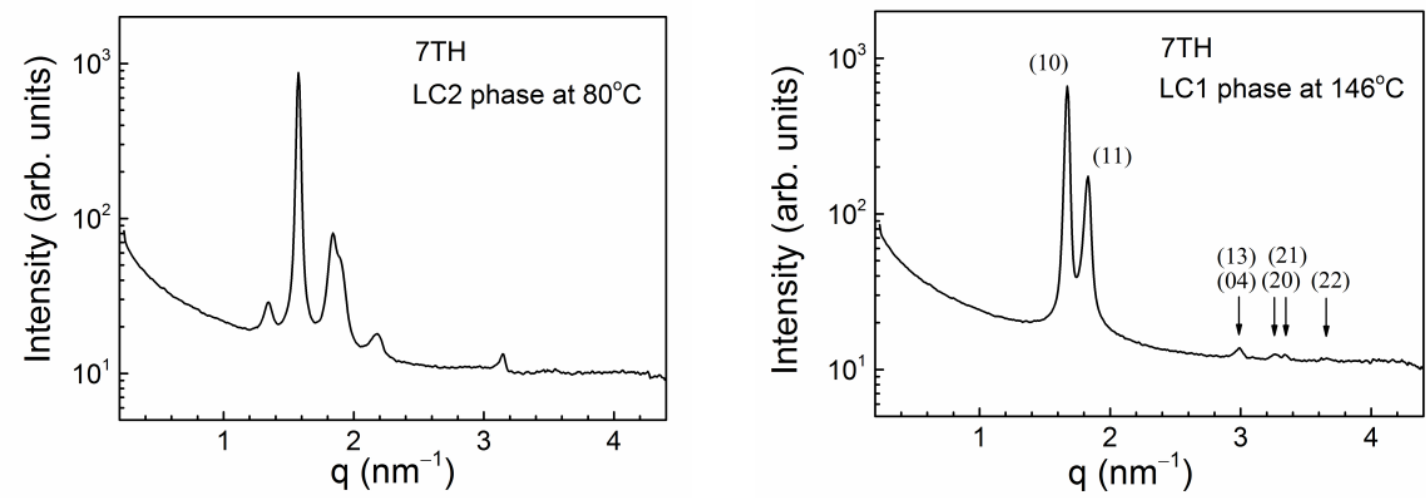

Figure 3. X-ray diffractograms of the two liquid crystal phases of 7TH.

Table 2. Peak positions for the two LC phases of 7TH (Fig. 3, where also the indexing of the LC1 phase is shown).

\begin{tabular}{|l|lll|ll|}
\hline peak number & \multicolumn{3}{|l|}{$7 \mathrm{TH}$ at $80^{\circ} \mathrm{C}(\mathrm{LC} 2)$} & \multicolumn{2}{l|}{$7 \mathrm{TH}$ at $146^{\circ} \mathrm{C}(\mathrm{LC} 1)$} \\
\hline & $\mathrm{q}_{\exp }\left(\mathrm{nm}^{-1}\right)$ & exp. ratio & theor. ratio & $\mathrm{q}_{\exp }\left(\mathrm{nm}^{-1}\right)$ & $\mathrm{q}_{\text {calc }}\left(\mathrm{nm}^{-1}\right)$ \\
\hline 1 & 1.34 & 1 & 1 & 1.67 & 1.67 \\
2 & 1.58 & 1.18 & 1.15 & 1.83 & 1.83 \\
3 & 1.86 & 1.39 & 1.53 & 2.99 & 3.01 \\
4 & 2.18 & 1.63 & 1.63 & $3.26 / 3.34$ & $3.34 / 3.43$ \\
5 & 3.14 & 2.34 & 2.31 & 3.66 & 3.67 \\
\hline
\end{tabular}

The LC1 phase at higher temperatures cannot be fitted to a cubic variant. However, the appearance is close to that reported for rectangular columnar phases. The assignment indicated in Fig. 3 leads to the numbers in the last column of Table 2 under LC1. We conclude to good agreement with a rectangular columnar phases with $a=3.76 \mathrm{~nm}, b=8.36 \mathrm{~nm}$ and disordered columns. A fit to a centred rectangular model is much less satisfactory. 


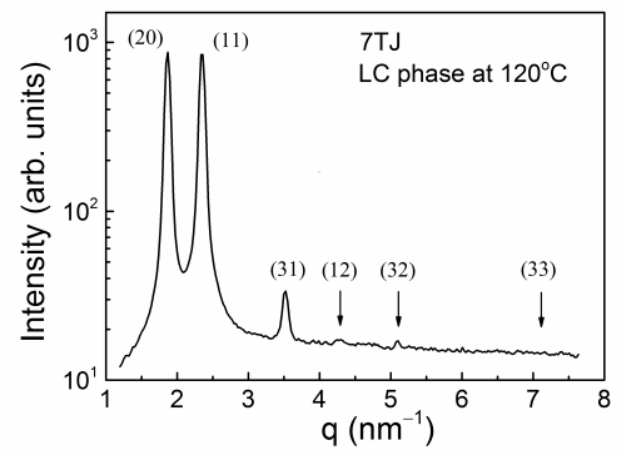

Figure 4. X-ray diffractogram of the single LC phase of 7TJ. The last peak at large $q$ is only visible in the two-dimensional picture.

Table 3. Peak positions for the LC phase of 7TJ (Fig. 4, where also the indexing is shown).

\begin{tabular}{|l|ll|}
\hline peak number & \multicolumn{2}{|l|}{$7 \mathrm{TJ}$ at $120^{\circ} \mathrm{C}$} \\
\hline & $q_{\exp }\left(\mathrm{nm}^{-1}\right)$ & $q_{\text {calc }}\left(\mathrm{nm}^{-1}\right)$ \\
\hline 1 & 1.86 & 1.86 \\
2 & 2.35 & 2.35 \\
3 & 3.52 & 3.53 \\
4 & 4.3 & 4.4 \\
5 & 5.1 & 5.1 \\
6 & 7.1 & 7.0 \\
\hline
\end{tabular}

Finally we come to the compound 7TJ for which a further increase in density of the alkyl chains occurs. We find over the whole temperature range below $146^{\circ} \mathrm{C}$ a single LC phase. The peak positions in the diffractogram of Fig. 4 cannot be fitted to any of the bicontinuous phases. The two main peaks are similar to the situation for the LC1 phase of 7TH. However, fitting to a rectangular columnar phase does not lead to good agreement, in particular not for the third main peak. This latter point is greatly improved taking a centred rectangular phase. From the comparison in Table 3 we conclude that such a phase with $a=6.72 \mathrm{~nm}$ and $b=2.93$ $\mathrm{nm}$ is a reasonable choice for the LC phase of 7TJ.

\section{Thin film results}

\subsection{AFM of monolayers}

Results from scanning force microscopy of monolayers are shown in Fig. 5. For 7T we reproduce the smooth rectangular shaped islands reported earlier. ${ }^{6}$ In addition, we note that these islands possess an in-plane structure with a period of $20 \mathrm{~nm}$. Submonolayers of 7TH and 7TJ display elongated holes associated with an in-plane supramolecular structure with a 
period of 3.4 and $3.6 \mathrm{~nm}$, respectively. Table 4 summarize the layer thickness inferred from the height profile. In the case of multilayers, for $7 \mathrm{~T}$ the adlayer is thinner than subsequent layers. However, the inverse occurs for $7 \mathrm{TH}$, i.e. the first layer is thicker than the next ones. For 7TJ no second layer thickness is available. Furthermore, for thick films, it becomes increasingly difficult to distinguish the different layers.

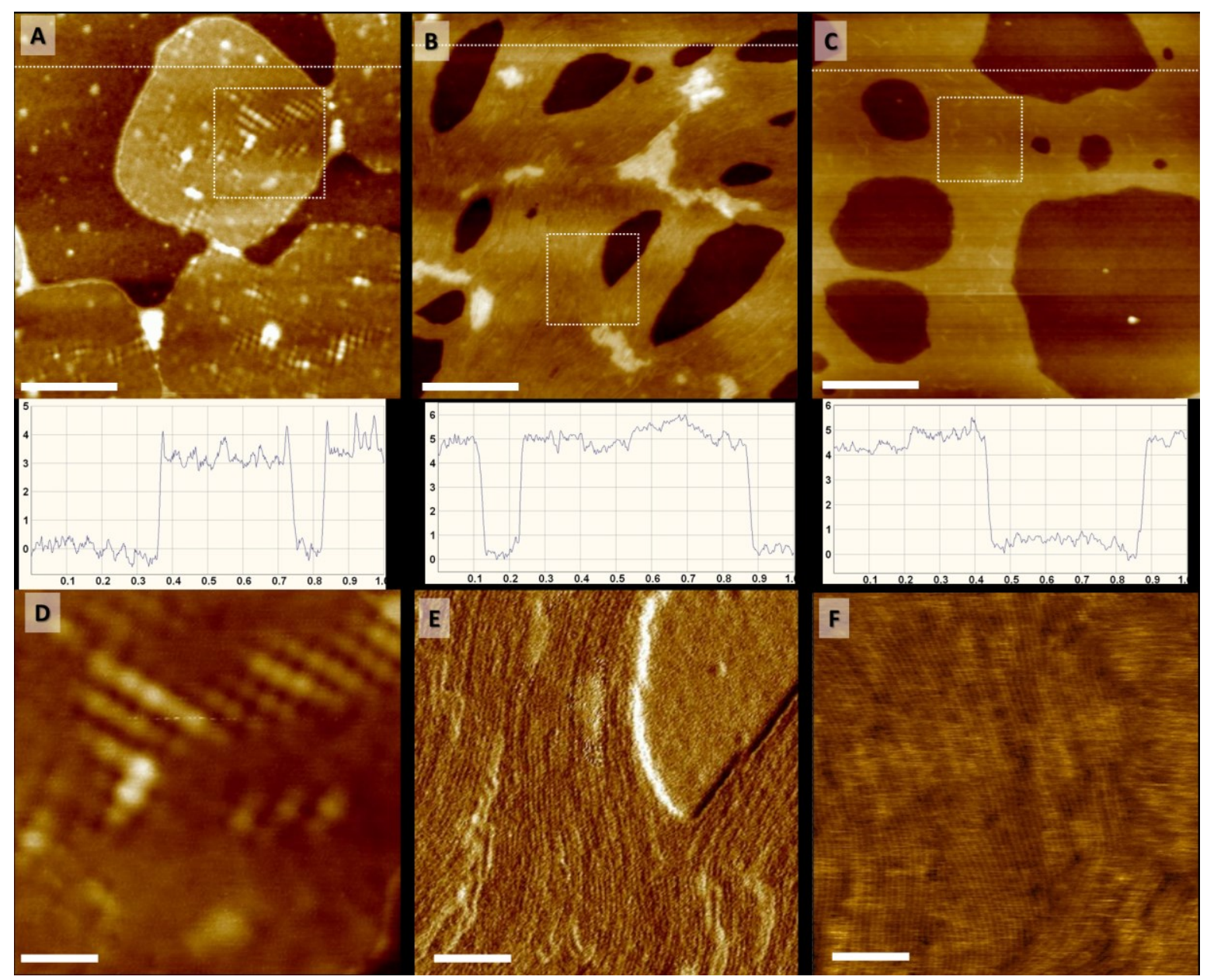

Figure 5. SFM height micrographs show the morphology of the submonolayer coverage depending on the molecular structure with the corresponding height profiles along the horizontal lines. (A) and (D) correspond to 7T, (B) and (E) to 7TH and (C) and (F) to 7TJ. The scale bars of the upper and lower row are $250 \mathrm{~nm}$ and $50 \mathrm{~nm}$, respectively. The lower row is selected areas in the larger scale images.

Table 4. Layer thickness $(\AA)$

\begin{tabular}{lll}
\hline & $\begin{array}{l}\text { XRR } \\
(\text { monolayer })\end{array}$ & $\begin{array}{l}\text { AFM } \\
\left(1^{\text {st }} / 2^{\text {nd }} \text { layer }\right)\end{array}$ \\
\hline $7 \mathrm{~T}$ & 37 & $34 / 38$ \\
$7 \mathrm{TH}$ & 54 & $49 / 36$ \\
$7 \mathrm{TJ}$ & 60 & 48 \\
\hline
\end{tabular}

\section{$3.2 X$-ray reflectivity of monolayers (XRR)}


Results from XRR are shown in Fig. S3 of the Supplementary Information. The data were fitted to a three-layer model with parameters as given in Table 5. In Table 4 the thicknesses of the monolayer as obtained from XRR is compared with AFM. The AFM values are somewhat smaller (as commonly observed), which is generally attributed to penetration of the AFM tip in the soft film.

From Table 5 we note that for $7 \mathrm{~T}$ the thickness of the middle layer is much smaller than the length of the septithiophene core $(26.7 \AA)$. This has been interpreted as a strongly tilted core structure. ${ }^{6}$ For $7 \mathrm{~T}$ the value is given by $\cos ^{-1}(12 / 26.7)=63^{\circ}$, larger than the value of $40^{\circ}$ reported earlier. ${ }^{6}$ Increasing the alkyl content leads to a much larger thickness of the middle layer of 7TH and 7TJ, approaching the thiophene core length. For all three compounds the core electron density is very similar, indicating clear separation of the thiophene and the alkylated parts of the molecules. In the order 7T, 7TH and 7TJ the thickness of the top and bottom layer increases as anticipated for larger alkyl content. A further point is the increase in electron density of the bottom layer from 7T via 7TH to 7TJ.

Table 5. Three-sublayer model parameters from XRR.

\begin{tabular}{lllll}
\hline \multirow{2}{*}{ Compound } & Model & $\begin{array}{l}\text { Thickness/ } \\
\AA\end{array}$ & $\begin{array}{l}\text { Electron density/ } \\
e \AA^{-3}\end{array}$ & $\begin{array}{l}\text { Roughness/ } \\
\AA\end{array}$ \\
\hline \multirow{3}{*}{$7 \mathrm{~T}$} & Alkyl (top layer) & 10 & 0.23 & 2 \\
& Thiophene core & 12 & 0.56 & 5 \\
& Alkyl (bottom layer) & 15 & 0.28 & 4 \\
$7 \mathrm{TH}$ & & & 2 \\
& Top layer & 12 & 0.17 & 5 \\
& Middle layer & 25 & 0.51 & 4 \\
& Bottom layer & 16 & 0.39 & 5 \\
$7 \mathrm{TJ}$ & & & & 4 \\
& Top layer & 18 & 0.27 & 0.4 \\
\hline
\end{tabular}

\subsection{Grazing-incidence diffraction of mono- and multilayers}

GIWAXS measurements (effectively determined by the electron-rich thiophene cores) are displayed in Fig. 6. For 7T the overall monolayer picture is rather similar as reported previously for substituted sexithiophenes. ${ }^{7}$ For the $7 \mathrm{~T}$ monolayer we find two main peaks assigned as $h k=11\left(q_{\perp}=10.82 \mathrm{~nm}^{-1}, q_{\mathrm{z}}=7.09 \mathrm{~nm}^{-1}\right)$ and $h k=20\left(q_{\perp}=7.2 \mathrm{~nm}^{-1}, q_{\mathrm{z}}=11.6 \mathrm{~nm}^{-1}\right)$. This is consistent with a centred rectangular lattice with $a=1.74 \mathrm{~nm}$ and $b=0.62 \mathrm{~nm}$. The tilt 
angle is given by ${ }^{29} \tan \theta=q_{z}^{20} / q_{\perp}^{20}=11.6 / 7.2$ or $\theta=58^{\circ}$, rather close to the XRR value of $63^{\circ}$. Using the total length of the thiophene core of $26.7 \AA$, the projection on the $\mathrm{z}$-axis then equals $26.7 \times \cos 58^{\circ}=14 \AA$. This compares reasonably well with the $12 \AA$ for the middle thiophene layer of 7T in XRR (see Table 5). The multilayer has essentially the same features as the monolayer, though more pronounced, and also more details like doubling of the peak around $q_{\perp}=6.2-6.5 \mathrm{~nm}^{-1}$ (see Fig. S4 of the Supplementary Information). However, note that the variation in $q_{\mathrm{rad}}=\left(q_{z}^{2}+q_{\perp}^{2}\right)^{1 / 2}$ is small: from $13.2 \mathrm{~nm}^{-1}$ for the (11)-peak of the monolayer to $14.4 \mathrm{~nm}^{-1}$ (average doublet) for the corresponding one of the multilayer.
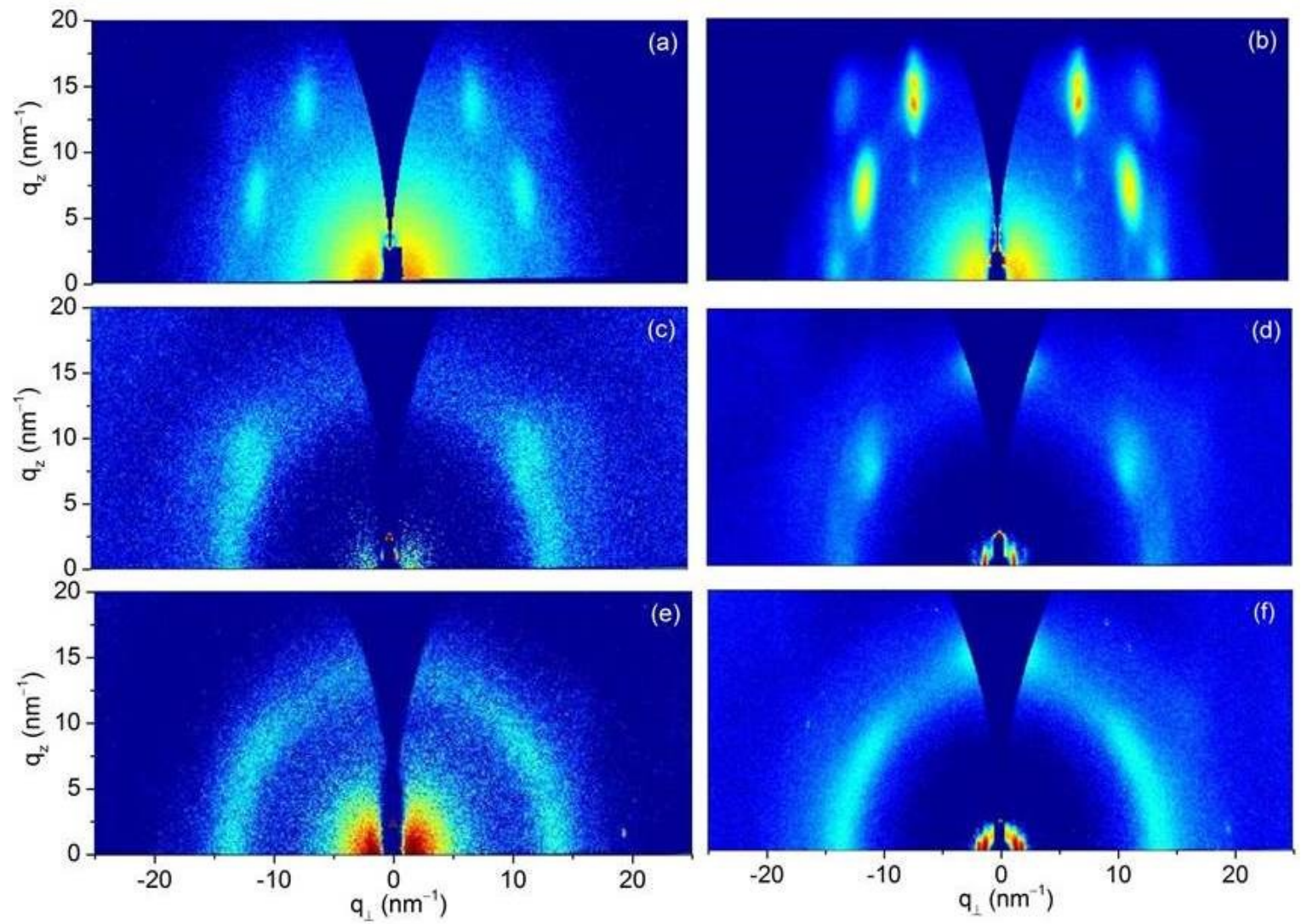

Figure 6. GIWAXS data for monolayer and three-layer films of 7T (a, b), 7TH (c, d) and 7TJ $(\mathrm{e}, \mathrm{f})$.

The essential features of the 7TH and 7TJ films are very similar. Both the monolayer and multilayer samples are dominated by a diffuse half-circular arc. However, note that the (11)-peak is still visible in all cases, though stronger for the multilayer samples. The diffuse semi-circle is characterized by $q_{\mathrm{rad}} \approx 13.6 \mathrm{~nm}^{-1}$ matching a periodicity of $2 \pi / 13.6=0.46 \mathrm{~nm}$, still similar as found for 7T. Evidently this number corresponds to an average lateral thiophene dimension, similar as observed in thiophene crystals. ${ }^{33}$ The samples are increasingly amorphous in the order 7T, 7TH, 7TJ. The multilayers differ qualitatively from the monolayers because of the presence of a (diffuse) peak along the vertical axis. The centre 
of these peaks at $q_{\mathrm{z}}=14.8 \mathrm{~nm}^{-1}$ (periodicity $0.42 \mathrm{~nm}$ ) is at a somewhat larger value than $q_{\text {rad }}$ of the diffuse arc.

Finally Fig. 7 shows a blow-up of the GIWAXS data at small angles. Interestingly, we observe for the multilayers some streaks in this region. For 7T these are arc-like along $q_{\perp}$, for 7TH and 7TJ parallel to the vertical z-axis. The latter can be interpreted as in-plane superstructures. The results for 7TH and 7TJ have been confirmed by GISAXS data (see Fig. S5 of the supplementary information for 7TJ). The associated periods are summarized in Table 6. The small ones of 7TH and 7TJ are close to the value of about $3.5 \mathrm{~nm}$ from AFM (Fig. 5).

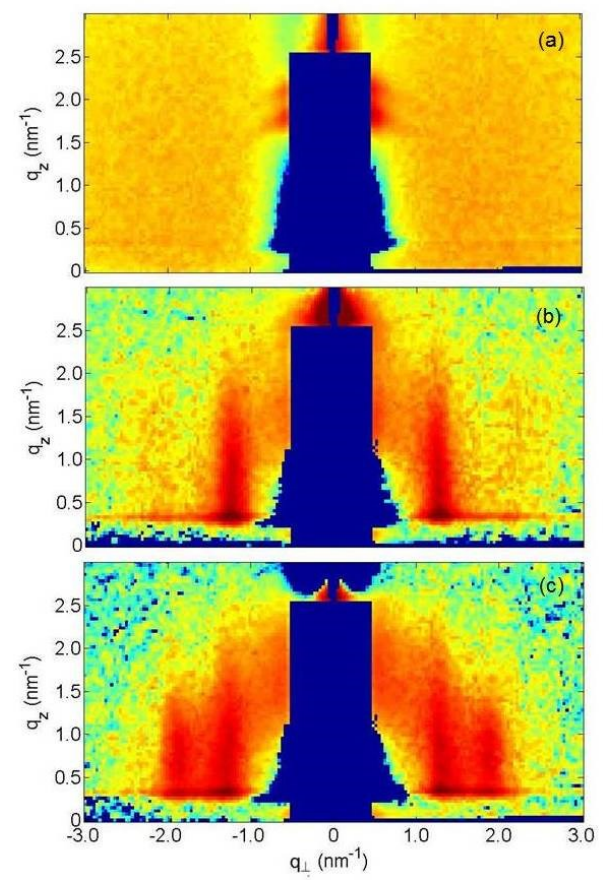

Figure 7. Small-angle GIWAXS pictures of the multilayer samples of 7T (a), 7TH (b) and 7TJ (c).

Table 6. Positions and periodicities of the small-angle peaks of Fig. 7.

\begin{tabular}{lllll}
\hline & $q\left(\mathrm{~nm}^{-1}\right)$ & $d(\mathrm{~nm})$ & $q\left(\mathrm{~nm}^{-1}\right)$ & $d(\mathrm{~nm})$ \\
\hline $7 \mathrm{~T}$ & $q_{\mathrm{z}}=1.81$ & 3.47 & $q_{\mathrm{z}}=2.11$ & 2.98 \\
$7 \mathrm{TH}$ & $q_{\perp}=1.26$ & 4.99 & $q_{\perp}=2.16$ & 2.91 \\
$7 \mathrm{TJ}$ & $q_{\perp}=1.3$ & 4.83 & $q_{\perp}=1.89$ & 3.32 \\
\hline
\end{tabular}

\section{Discussion}

The three compounds studied differ considerably in the size of the end groups. In terms of the bulkiness parameter $P$ introduced by Kreyes et al. ${ }^{24}$ - a measure of the volume ratio of branched part and linear spacer of the end groups - our bulky compounds have values $P=67$ (7TH) and $P=367$ (7TJ), respectively. The increase reflects the larger amount of alkyl chains 
incorporated in the packing. Tschierske and co-workers ${ }^{31,34,35}$ have studied the increase of alkyl content for chains attached laterally to quaterthiophene cores. Though different from our situation of end chains, their observation of transitions smectic $\leftrightarrow$ square columnar $\leftrightarrow$ bicontinuous fits into the trend mentioned already. ${ }^{30-32}$. In their situation the walls of the cylinders are formed by a couple of aromatic quaterthiophene cores. The work of Yasuda et al. ${ }^{32}$ on sexithiophenes with wedge-shaped end substituents is closer to our situation. In the order of increasing alkyl content they find the following LC phases: $\mathrm{Sm}+\mathrm{Col}_{\text {hex }}, \mathrm{Sm}+\mathrm{Col}_{\text {square }}$ and $\mathrm{Col}_{\text {square }}+$ Cubic. They assume that approximately 3-4 molecules self-assemble into an overall disk-like stratum and successively stack to one another to form supramolecular columns. No further model for the assembling of these 3-4 molecules was given. We assume that in our case optimum $\pi-\pi$ interactions can be retained if the long thiophene cores are packed on top of each other with additionally some relative rotations of their long axis. In this way a compromise can be reached between creating space for the alkyl chains and keeping some $\pi-\pi$ coupling.

A tentative model for the packing of 7TJ, shown in Fig. 8, was obtained using molecular mechanics calculations $(\mathrm{MM}+)$. The geometry of one molecule 7TJ in the gas phase was optimized and five further copies of this molecule added manually, keeping a certain $\pi-\pi$ overlap and avoiding steric conflicts of the bulky alkyl chains. This produces in a natural way a twisted columnar arrangement - in agreement with our assumption of rotation of the thiophene cores - leading potentially to a helical structure. Full helicity has not been observed, but such structural elements might still be present on a short-range scale. In that situation the structure of Fig. 8 still provides a natural basis for a columnar phase. In the mean time we performed molecular dynamics calculations confirming the formation of a columnar structure. This would be too much to be included here and will be published separately. The transition from columnar to bicontinuous cubic requires a further restructuring of the thiophene cores and the aliphatic chains.
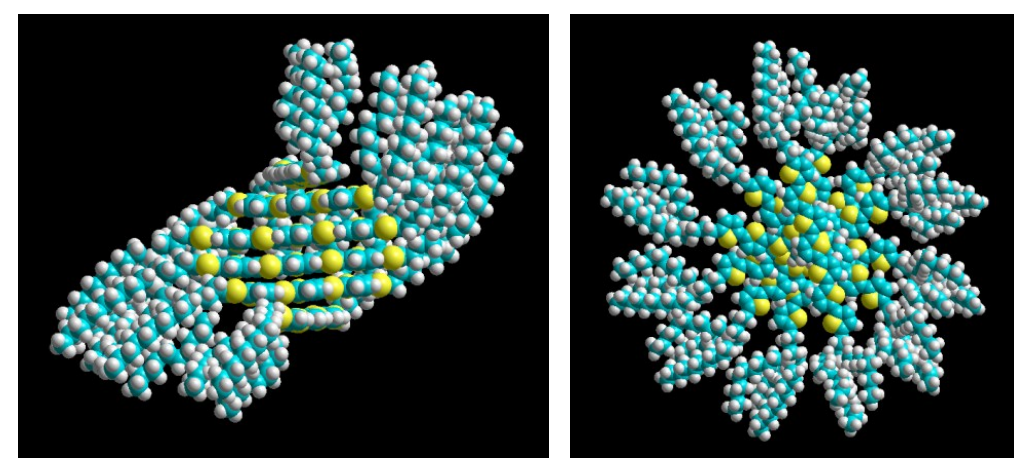

Figure 8. Suggested packing of 7TJ. (a) Side view indicating a pitch of about $3 \mathrm{~nm}$. (b) Top view with a diameter of about $5 \mathrm{~nm}$. 
The GIWAXS of the 7T monolayer can be interpreted along the same lines as previously established for terminally substituted thiophenes. ${ }^{7}$ Using the theory of Kaganer et al. ${ }^{36}$ this leads to the model of Fig. 9 with a tilt angle of the thiophene core of $58^{\circ}$. In this way the different cross sections of the thiophene core and the alkyl group can be accommodated. This result is qualitatively similar as reported earlier for $7 \mathrm{~T}^{6}$ The GIWAXS of the multilayer is rather similar to the monolayer. We attribute the doubling of the peaks to a different tilt angle in the layers not directly at the substrate. Making this quantitative, attributing the 20refelction to either the upper or the lower peak would a variation of $2^{\circ}$ in the tilt angle of the core. Similar variations with the distance to the substrate have been reported by Mannebach et al. for dihexylsexithiophene. ${ }^{37}$ The superstructure may be the result of two-dimensional organization of the molecules. In a monolayer, the interfacial forces and lack of interdigitation of the alkyl chains leads to a large tilt angle, thereby weakening the $\pi-\pi$ interactions. The proposed molecular tilt reduces the steric constraints and solves the packing problem along one axis of the layer plane, but not along the second axis. We believe a more elaborated model is needed that takes into account the two-dimensional geometric requirements that could be the cause of this superstructure.

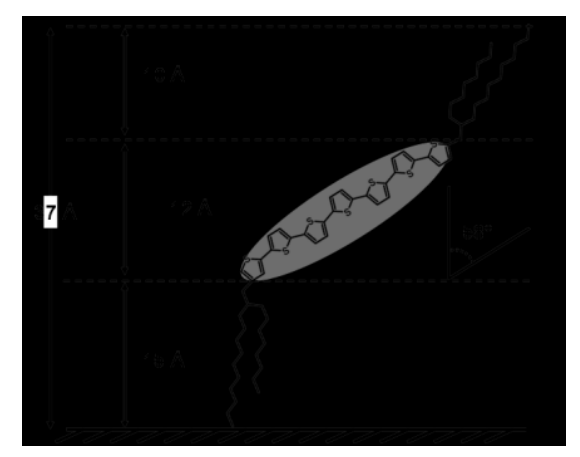

Figure 9. Model for the monolayer of 7T using the actual XRR layer thicknesses and the tilt angle from GIWAXS.

The monolayers of 7TH and 7TJ are increasingly amorphous. The corresponding multilayers are qualitatively different in terms of an additional strong peak along the z-axis. The difference can in principle be understood from the relief from constraints by the substrate for the multilayer. Turning to the bulk, we note that a bicontinuous cubic phase is difficult to translate into a monolayer structure. However, let us recall the tendency smectic $\rightarrow$ columnar $\leftrightarrow$ bicontinuous cubic upon increasing the alkyl content of a LC compounds. The latter transition often involves a square columnar phase. ${ }^{32}$ Such a phase might be a good choice if we look for elements from the bulk structure of 7TH and/or 7TJ most compatible to a 
monolayer (or thin multilayer). This idea is schematically drawn in Fig. 10, and is supported by the results from XRR. Averaging the model over the xy-plane would indeed give a dense middle layer with a thickness of the order of the septithiophene core length. In agreement with the model of Fig. 8, we expect the complexed thiophene core to rotate along the column axes (short-range only). Such a structure would provide an optimal compromise between retaining some $\pi-\pi$ interactions and creating space for the bulky alkyl groups.

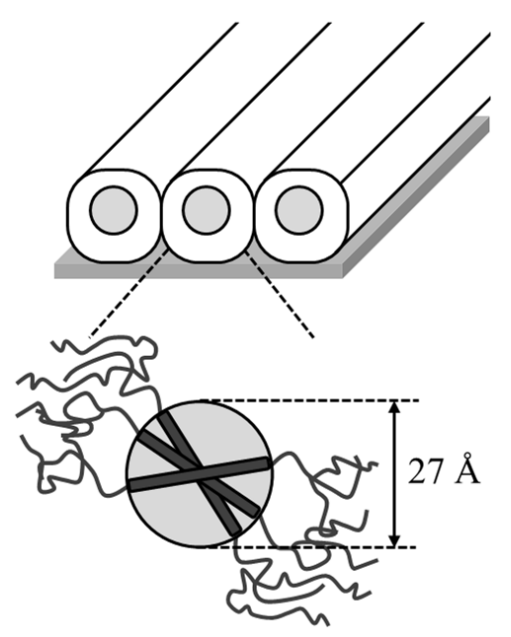

Figure 10. Proposed structural model for the monolayer films of 7TH and/or 7TJ. Note that we did not try to include further anisotropies like a possible flatter columnar nanostructure. The lower part of the figure is a partial projection of the thiophene cores rotated along the column axis.

In the model as proposed the column axis is a direction of disorder. The thickness of the monolayer and the lateral period should be of the same order of magnitude, which is approximately (but not precisely) as observed (around $4 \mathrm{~nm}$ ). The crystallization of the thiophene cores in the monolayer can indeed be expected to be weak. In the model of Fig. 10 alkyl edges surround the thiophene column centres. Perpendicular to the columns (horizontal direction in Fig. 6) the number of thiophenes grouped together is expected to vary depending on the amount of alkyl chains to be packed, which could induce differences between 7TH and 7TJ. Note that Fig. 9 (model for 7T) and Fig. 10 (with small modifications the proposed model for 7TH and 7TJ) differ little regarding the average electron density along the layer normal, in agreement with the XRR results. Furthermore, the symmetry might be well disturbed by each of the two interfaces. This process could introduce some asymmetry normal to the monolayer, which is very pronounced for the bottom layer of 7TJ (see Table 4).

We have started with emphasizing the short-range order, which is observed at a similar scattering vector as for the (11)-peak (distance of closest approach in the centred rectangular 7T lattice). To account for the peaks observed, we need to modify the (almost isotropic) 
model of Fig. 10 introducing some preferences for the direction of the thiophene cores with respect to the substrate to explain the observed changes in the scattering signal. The (11)peaks indicate that the tilt angle of about $60^{\circ}$ (that leads to an approximate match of the cross sections of thiophene and alkyl) still has some preference. For the 7TJ monolayer this is less true and a wider distribution of tilts is observed. The strong peak along $q_{z}$ observed in GIWAXS of the three-layer film of 7TH indicates correlations inside and/or between the columns, leading to some crystallization of the thiophene cores. Evidently there is some preference for ordering of the conjugated cores with respect to the layer normal, which is easier for the second and third layer, not constrained by the substrate. In spite of the extensive measurements the frustrated system escapes from more precise modelling.

Finally we note that the SAXS data (Fig. 7) give a series of periods of which the smaller ones for 7TH and 7TJ are compatible with the XRR and AFM values. These are roughly in agreement with the length of the molecules or the diameter of the columns. We have observed an increased tendency to form superstructures in systems with longer thiophene cores (T9, $\mathrm{T} 11$ ), indicating an escape from increasing frustration. The arcs observed for $7 \mathrm{~T}$ in Fig. 7a are probably due to a non-planar surface. 


\section{Conclusions and outlook}

In conclusion we have compared the structure and phase behaviour of three alkylsubstituted septithiophenes with increasing dimensions of the alkyl groups. For a larger alkyl volume we observe a phase sequence smectic $\leftrightarrow$ columnar/bicontinuous cubic. In monolayer films, the packing constraints have to be combined with limiting surfaces. Moreover, for 7TH the bicontinuous cubic phase observed in the bulk cannot be supported in monolayer films. We propose a monolayer structure of columns parallel to the substrate, which may have shortrange helical order along the columns. This situation allows for some $\pi-\pi$ interactions of the thiophenes cores in spite of the packing constraints of the bulky alkyl groups. For multilayer films these constraints are somewhat relaxed leading to specific tilt distributions giving weak $\mathrm{X}$-ray peaks.

The progress in the synthesis of substituted thiophenes of different core length, ${ }^{15-17}$ allows in the future a further evaluation of the intricate problems of packing and frustration in anisotropic conjugated systems.

\section{Acknowledgement}

This work was supported by the German Science Foundation Projects Structuring SemiConducting (Ultra)thin MO982/2-3. We thank Matthieu Defaux for his earlier X-ray work on the bulk samples and Andreas Kreyes for his contribution to the synthesis of the materials. We are grateful to Jan Perlach for his expert help at beamline WB4 (Hasylab, Hamburg) and to Zhihua Hong for his share in the synchrotron experiments. 


\section{Experimental}

Materials. The oligothiophenes $7 \mathrm{~T},{ }^{15} 7 \mathrm{TH}^{24}$ and $7 \mathrm{TJ}^{17}$ were prepared according to the literature given.

Differential scanning calorimetry. (Differential scanning calorimetry (DSC) was performed on a Perkin-Elmer DSC 7 with a heating rate of $10 \mathrm{Kmin}^{-1}$.

Optical microscopy. The Polarising optical microscopy (POM) images were taken between two glass slides on a Zeiss Axioskop equipped with a Mettler FP 90 hot stage.

Molecular calculations. The molecular model was constructed from 6 molecules by the program Hyperchem 7.5 (Hypercube, Inc. 2002). At first one molecule was geometry optimized with molecular mechanics (MM2) in the gas phase and then 6 copies of that molecule were manually arranged with a spacing of the aromatic planes of ca. $3.5 \AA$ and the branched substituents avoiding any steric conflict.

SFM. Scanning Force Microscopy was done in the tapping mode (NanoScope V, Digital Instruments Veeco Instruments, Santa Barbara, CA) under ambient conditions. The standard silicon cantilevers used had a spring constant of $5 \sim 37 \mathrm{~N} / \mathrm{m}$ and an oscillation frequency of $\sim 75 \mathrm{kHz}$. Data were processed using Digital NanoScope Analysis software, version 1.12. Bulk X-ray scattering. XRD pattern of extruded fibres were obtained at various temperatures. The experiments were done at beamline ID02 of the ESRF (Grenoble, France) at 10.keV using a 2D photon-counting pixel detector. More details can be found in Ref. 5 . XRR. X-Ray Reflectivity of films was performed in-house using an Empyrean setup from PANalytical using $\mathrm{Cu} K_{\alpha}$ radiation. A parabolic graded multilayer system converted the divergent line source $(12 \times 0.04 \mathrm{~mm})$ into an almost parallel beam with divergence $<0.03^{\circ}$. After reflection from the sample the intensity was collected by a pixel detector $(256 \times 256$ pixels of $55 \mu \mathrm{m}$, all connected as a $0-\mathrm{D}$ detector) as a function of the scattering vector $q_{z}=(4 \pi / \lambda) \sin \theta, 2 \theta$ being the scattering angle. The latter was fixed at twice the incident angle $\theta$, which sets $q_{z}$ along the film normal. The experimental data were model-independent fitted using the program STOCHFIT which slices the film using a large number of thin layers. Subsequently the resulting density profile was analyzed in terms a three-layer internal structure of the film.

GID. Grazing-Incidence Diffraction (GIWAXS and GISAXS) was performed at beamline BW4 of Hasylab (DESY, Hamburg). ${ }^{38}$ The sample-detector distance was varied depending on $q$-range chosen (GIWAXS or GISAXS), while the incident angle was set to $0.2^{\circ}$. The scattering signal was recorded by a 2D detector (MAR CCD, $2048 \times 2048$ pixels). Image conversion was done using the program GIXSGUI. ${ }^{39}$ 
References

1. Goodby, G. W. C., P. J.; Kato,T.; Tschierske, C.; Gleeson, H. F.; Raynes, E. P. (Editors), Handbook of Liquid Crystals, 2nd edition. Wiley-VCH: Weinheim, 2014.

2. Pelzl, G.; Diele, S.; Weissflog, W., Banana-Shaped Compounds-A New Field of Liquid Crystals. Advanced Materials 1999, 11, (9), 707-724.

3. Reddy, R. A.; Tschierske, C., Bent-core liquid crystals: polar order, superstructural chirality and spontaneous desymmetrisation in soft matter systems. Journal of Materials Chemistry 2006, 16, (10), 907-961.

4. Fichou, D., Handbook of Oligo- and Polythiophenes. WILEY-VCH Verlag GmbH: 1999.

5. Parrish, R. M.; Sherrill, C. D., Quantum-Mechanical Evaluation of $\pi-\pi$ versus Substituent $-\pi$ Interactions in $\pi$ Stacking: Direct Evidence for the Wheeler-Houk Picture. Journal of the American Chemical Society 2014, 136, (50), 17386-17389.

6. Defaux, M.; Gholamrezaie, F.; Wang, J.; Kreyes, A.; Ziener, U.; Anokhin, D. V.; Ivanov, D. A.; Moser, A.; Neuhold, A.; Salzmann, I.; Resel, R.; de Leeuw, D. M.; Meskers, S. C. J.; Moeller, M.; Mourran, A., Solution-Processable Septithiophene Monolayer Transistor. Advanced Materials 2012, 24, (7), 973-978.

7. Wang, J.; de Jeu, W. H.; Ziener, U.; Polinskaya, M. S.; Ponomarenko, S. A.; Ruecker, U.; Ruderer, M. A.; Herzig, E. M.; Müller-Buschbaum, P.; Moeller, M.; Mourran, A., Monolayer Properties of Asymmetrically Substituted Sexithiophene. Langmuir 2014, 30, (10), 2752-2760.

8. Al-Mahboob, A.; Fujikawa, Y.; Sadowski, J. T.; Hashizume, T.; Sakurai, T., Formation of giant crystalline grain via delayed growth process driven by organic molecular anisotropy. Physical Review B 2010, 82, (23), 235421.

9. Anthony, J. E., Functionalized Acenes and Heteroacenes for Organic Electronics. Chemical Reviews 2006, 106, (12), 5028-5048.

10. Forrest, S. R., The path to ubiquitous and low-cost organic electronic appliances on plastic. Nature 2004, 428, (6986), 911-918.

11. Lu, M.; Nagamatsu, S.; Yoshida, Y.; Chikamatsu, M.; Azumi, R.; Yase, K., Solutionprocessable Oligothiophene Derivatives with Branched Alkyl Chains and Their Thin-film Transistor Characteristics. Chemistry Letters 2010, 39, (1), 60-61.

12. O’Neill, M.; Kelly, S., Liquid Crystals for Organic Field-Effect Transistors. In Liquid Crystalline Semiconductors, Bushby, R. J.; Kelly, S. M.; O'Neill, M., Eds. Springer Netherlands: 2013; Vol. 169, pp 247-268.

13. Sirringhaus, H., 25th Anniversary Article: Organic Field-Effect Transistors: The Path Beyond Amorphous Silicon. Advanced Materials 2014, 26, (9), 1319-1335.

14. Dinelli, F.; Murgia, M.; Levy, P.; Cavallini, M.; Biscarini, F.; de Leeuw, D. M., Spatially Correlated Charge Transport in Organic Thin Film Transistors. Physical Review Letters 2004, 92, (11), 116802.

15. Ellinger, S.; Kreyes, A.; Ziener, U.; Hoffmann-Richter, C.; Landfester, K.; Möller, M., Aggregation Phenomena of Long $\alpha$ - and $\alpha, \omega$-Substituted Oligothiophenes - the Effect of Branched vs. Linear End-Groups. European Journal of Organic Chemistry 2007, 2007, (34), 5686-5702.

16. Ellinger, S.; Ziener, U.; Thewalt, U.; Landfester, K.; Möller, M., Synthesis and SelfOrganization of $\alpha, \omega$-Substituted Oligothiophenes with Long, Branched Alkyl Substituents. Chemistry of Materials 2007, 19, (5), 1070-1075.

17. Kreyes, A.; Amirkhani, M.; Lieberwirth, I.; Mauer, R.; Laquai, F.; Landfester, K.; Ziener, U., The Longest $\beta$-Unsubstituted Oligothiophenes and Their Self-Assembly in Solution. Chemistry of Materials 2010, 22, (23), 6453-6458. 
18. Leroy, J.; Boucher, N.; Sergeyev, S.; Sferrazza, M.; Geerts, Y. H., Symmetrical and Nonsymmetrical Liquid Crystalline Oligothiophenes: Convenient Synthesis and TransitionTemperature Engineering. European Journal of Organic Chemistry 2007, 2007, (8), 12561261.

19. Murphy, A. R.; Fréchet, J. M. J.; Chang, P.; Lee, J.; Subramanian, V., Organic Thin Film Transistors from a Soluble Oligothiophene Derivative Containing Thermally Removable Solubilizing Groups. Journal of the American Chemical Society 2004, 126, (6), 1596-1597. 20. Funahashi, M.; Hanna, J. I., High Carrier Mobility up to $0.1 \mathrm{~cm} 2 \mathrm{~V}-1 \mathrm{~s}-1$ at Ambient Temperatures in Thiophene-Based Smectic Liquid Crystals. Advanced Materials 2005, 17, (5), 594-598.

21. Melucci, M.; Favaretto, L.; Bettini, C.; Gazzano, M.; Camaioni, N.; Maccagnani, P.; Ostoja, P.; Monari, M.; Barbarella, G., Liquid-Crystalline Rigid-Core Semiconductor Oligothiophenes: Influence of Molecular Structure on Phase Behaviour and Thin-Film Properties. Chemistry - A European Journal 2007, 13, (36), 10046-10054.

22. Meng, Q.; Sun, X.-H.; Lu, Z.; Xia, P.-F.; Shi, Z.; Chen, D.; Wong, M. S.; Wakim, S.; Lu, J.; Baribeau, J.-M.; Tao, Y., Syntheses, Phase Behavior, Supramolecular Chirality, and Field-Effect Carrier Mobility of Asymmetrically End-Capped Mesogenic Oligothiophenes. Chemistry - A European Journal 2009, 15, (14), 3474-3487.

23. Kreyes, A.; Ellinger, S.; Landfester, K.; Defaux, M.; Ivanov, D. A.; Elschner, A.; Meyer-Friedrichsen, T.; Ziener, U., Fine Tuning of Solid-State Properties of Septithiophenes by Tailoring the Substituents. Chemistry of Materials 2010, 22, (6), 2079-2092.

24. Kreyes, A.; Mourran, A.; Hong, Z.; Wang, J.; Möller, M.; Gholamrezaie, F.; Roelofs, W. S. C.; de Leeuw, D. M.; Ziener, U., Predictability of Thermal and Electrical Properties of End-Capped Oligothiophenes by a Simple Bulkiness Parameter. Chemistry of Materials 2013, $25,(10), 2128-2136$.

25. Al-Mahboob, A.; Sadowski, J. T.; Fujikawa, Y.; Nakajima, K.; Sakurai, T., Kineticsdriven anisotropic growth of pentacene thin films. Physical Review B 2008, 77, (3), 035426.

26. Garnier, F.; Yassar, A.; Hajlaoui, R.; Horowitz, G.; Deloffre, F.; Servet, B.; Ries, S.; Alnot, P., Molecular engineering of organic semiconductors: design of self-assembly properties in conjugated thiophene oligomers. Journal of the American Chemical Society 1993, 115, (19), 8716-8721.

27. McCulloch, I.; Heeney, M.; Bailey, C.; Genevicius, K.; MacDonald, I.; Shkunov, M.; Sparrowe, D.; Tierney, S.; Wagner, R.; Zhang, W.; Chabinyc, M. L.; Kline, R. J.; McGehee, M. D.; Toney, M. F., Liquid-crystalline semiconducting polymers with high charge-carrier mobility. Nat Mater 2006, 5, (4), 328-333.

28. van Breemen, A. J. J. M.; Herwig, P. T.; Chlon, C. H. T.; Sweelssen, J.; Schoo, H. F. M.; Setayesh, S.; Hardeman, W. M.; Martin, C. A.; de Leeuw, D. M.; Valeton, J. J. P.; Bastiaansen, C. W. M.; Broer, D. J.; Popa-Merticaru, A. R.; Meskers, S. C. J., Large Area Liquid Crystal Monodomain Field-Effect Transistors. Journal of the American Chemical Society 2006, 128, (7), 2336-2345.

29. Li, L.; Séréro, Y.; Koch, M. H. J.; de Jeu, W. H., Microphase Separation and Crystallization in an Asymmetric Diblock Copolymer: Coupling and Competition. Macromolecules 2003, 36, (3), 529-532.

30. Hori, R.; Miwa, Y.; Yamamoto, K.; Kutsumizu, S., Phase Structure and Phase Transition Mechanism for Light-Induced Ia3d Cubic Phase in 4'-n-Docosyloxy-3'nitrobiphenyl-4-carboxlic acid/Ethyl 4-(4'-n-docosyloxyphenylazo)benzoate Binary Mixture. The Journal of Physical Chemistry B 2014, 118, (13), 3743-3749.

31. Tschierske, C., Liquid crystal engineering - new complex mesophase structures and their relations to polymer morphologies, nanoscale patterning and crystal engineering. Chemical Society Reviews 2007, 36, (12), 1930-1970. 
32. Yasuda, T.; Ooi, H.; Morita, J.; Akama, Y.; Minoura, K.; Funahashi, M.; Shimomura, T.; Kato, T., $\pi$-Conjugated Oligothiophene-Based Polycatenar Liquid Crystals: SelfOrganization and Photoconductive, Luminescent, and Redox Properties. Advanced Functional Materials 2009, 19, (3), 411-419.

33. Fichou, D., Structural order in conjugated oligothiophenes and its implications on opto-electronic devices. Journal of Materials Chemistry 2000, 10, (3), 571-588.

34. Gao, H.; Ye, Y.; Kong, L.; Cheng, X.; Prehm, M.; Ebert, H.; Tschierske, C., Dithiophene based X-shaped bolaamphiphiles: liquid crystals with single wall honeycombs and geometric frustration. Soft Matter 2012, 8, (42), 10921-10931.

35. Prehm, M.; Götz, G.; Bäuerle, P.; Liu, F.; Zeng, X.; Ungar, G.; Tschierske, C., Complex Liquid-Crystalline Superstructure of a $\pi$-Conjugated Oligothiophene. Angewandte Chemie International Edition 2007, 46, (41), 7856-7859.

36. Kaganer, V. M.; Peterson, I. R.; Kenn, R. M.; Shih, M. C.; Durbin, M.; Dutta, P., Tilted phases of fatty acid monolayers. The Journal of Chemical Physics 1995, 102, (23), 9412-9422.

37. Mannebach, E. M.; Spalenka, J. W.; Johnson, P. S.; Cai, Z.; Himpsel, F. J.; Evans, P. G., High Hole Mobility and Thickness-Dependent Crystal Structure in $\alpha, \omega-$ Dihexylsexithiophene Single-Monolayer Field-Effect Transistors. Advanced Functional Materials 2013, 23, (5), 554-564.

38. Perlich, J.; Rubeck, J.; Botta, S.; Gehrke, R.; Roth, S. V.; Ruderer, M. A.; Prams, S. M.; Rawolle, M.; Zhong, Q.; Körstgens, V.; Müller-Buschbaum, P., Grazing incidence wide angle x-ray scattering at the wiggler beamline BW4 of HASYLAB. Review of Scientific Instruments 2010, 81, (10), 105105.

39. Jiang, Z., GIXSGUI: a MATLAB toolbox for grazing-incidence X-ray scattering data visualization and reduction, and indexing of buried three-dimensional periodic nanostructured films. J Appl Crystallogr 2015, 48, 917-926. 
Table of Content: TOC

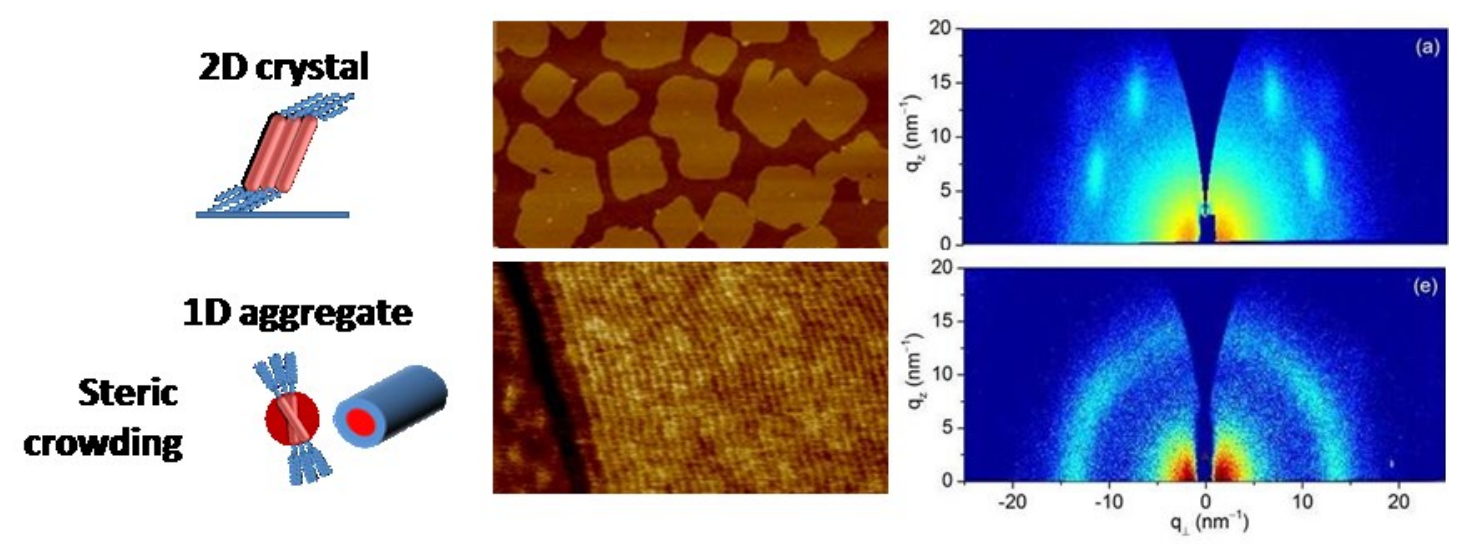

